PROCEEDINGS OF THE

AMERICAN MATHEMATICAL SOCIETY

Volume 132, Number 2, Pages 341-346

S 0002-9939(03)07085-0

Article electronically published on September 5, 2003

\title{
TIGHTLY CLOSED IDEALS OF SMALL TYPE
}

\author{
ADELA VRACIU \\ (Communicated by Bernd Ulrich)
}

\begin{abstract}
We study the smallest possible type of tightly closed ideals that are cofinal with the powers of the maximal ideal; this numerical invariant yields information about the tight closure of arbitrary ideals in the ring.
\end{abstract}

\section{INTRODUCTION}

Tight closure was introduced by Hochster and Huneke in [HH1], and it is an operation defined on the ideals of $R$, where $(R, \mathfrak{m})$ is a Noetherian local ring of characteristic $p>0$. We will use $q$ to denote a power of $p, q=p^{e}$.

1.1. Definition. Let $I$ be an ideal of $R$. An element $x \in R$ is in the tight closure of $I$, denoted $I^{*}$, if there exists a $c \in R$ not in any minimal prime, such that $c x^{q} \in I^{[q]}$ for every $q=p^{e} \gg 0$, where $I^{[q]}=\left(i^{q} \mid i \in I\right)$.

Although the element $c$ in the definition may a priori depend on $I$, there are elements which can be used to test tight closure for all the ideals $I$ (provided that the ring satisfies certain mild conditions, such as reduced and essentially of finite type over an excellent local ring); see [HH2]. In this paper we will always assume that the ring under consideration admits test elements.

1.2. Definition. An element $c \in R$ not in any minimal primes is a test element if $c x \in I$ for every ideal $I$ and every $x \in I^{*}$. The ideal $\tau$ generated by all the test elements is called the test ideal.

Due to the fact that the definition requires infinitely many tests, there are no known algorithms for computing tight closure, except in very special cases. An equivalent problem is finding all the tightly closed ideals in a ring $R$. In this paper, we restrict attention to the case of $\mathfrak{m}$-primary ideals, and we search for $\mathfrak{m}$-primary tightly closed ideals of the "smallest possible" type. We recall a few relevant facts about the type of $\mathfrak{m}$-primary ideal.

1.3. Definition. Let $I$ be an $\mathfrak{m}$-primary ideal. The type of $I$ is the vector space dimension of $(I: \mathfrak{m}) / I$ over $R / \mathfrak{m}$.

1.4. Observation. 1 . The m-primary irreducible ideals have type 1 .

2. An $\mathfrak{m}$-primary ideal $I$ has type $k$ if and only if it can be written as an irredundant intersection of $k$ irreducible ideals.

Received by the editors July 8, 2002 and, in revised form, October 9, 2002.

2000 Mathematics Subject Classification. Primary 13A35.

Key words and phrases. Tight closure, type. 
3. Any m-primary ideal of type $\geq k$ can be obtained as an intersection of ideals of type $k$.

4. Any intersection of tightly closed ideals is tightly closed.

Thus, once we know the tightly closed ideals of smallest possible type, we can get tightly closed ideals of larger type by taking intersections.

This approach raises the question of what the "smallest possible" type of tightly closed ideals is; this "smallest" type will be denoted $t(R)$ (see Definition [2.4). We show that an upper bound for $t(R)$ is provided by the number of generators of the test ideal $\tau$, which is denoted by $\nu(\tau)$ (see Observation 2.6). We then ask what are the implications of knowing $t(R)$ in terms of obtaining information about the tight closure of arbitrary ideals in $R$. Our main result is Theorem 3.1, which shows that knowing that a certain ideal is tightly closed has implications concerning the tight closure of all ideals that contain it. In light of this result, it becomes desirable to impose the condition that the tightly closed ideals of "smallest" type are cofinal with the powers of $\mathfrak{m}$, so that any $\mathfrak{m}$-primary ideal contains one such ideal.

\section{BACKGROUND AND DEFINITIONS}

The results of this paper draw on and extend results coming from three different directions:

1. the characterization of weakly F-regular rings as those rings in which there exist irreducible tightly closed ideals cofinal with the powers of the maximal ideal ([HH1]),

2. results related to the existence and properties of strong test ideals $([\mathrm{Hu}],[\mathrm{Vr}])$,

3. results about big ideals ([HHV]).

We recall the relevant definitions and results from $[\mathrm{Hu}]$ and $[\mathrm{Vr}$ :

2.1. Definition. If $R$ is a characteristic $p$ Noetherian ring, an ideal $T \subset R$ is called a strong test ideal if it has the property that $T I^{*}=T I$ for every ideal $I \subset R$.

2.2. Theorem $([\mathrm{Hu}])$. If $R$ has a strong test ideal $T$ and $k$ is the number of generators of $T$, then $\left(I^{*}\right)^{k} \subseteq I$ for every ideal $I \subset R$.

2.3. Theorem $([\mathrm{Vr})$. Let $(R, \mathfrak{m})$ be a characteristic $p$ complete local ring with test ideal $\tau$. Then for every ideal $I \subset R$, the ideal $I: \tau$ is tightly closed. In particular, this implies that $\tau$ is a strong test ideal.

This result is also valid whenever the test ideal commutes with completion, e.g. for $\mathbf{Q}$-Gorenstein rings, and for graded rings.

The two numerical invariants introduced in this paper are the following:

\subsection{Definition.}

$$
\begin{aligned}
c(R) & :=\inf \left\{k \mid\left(I^{*}\right)^{k} \subseteq I \text { for every ideal } I \subseteq R\right\} \\
& =\inf \left\{k \mid\left(I^{*}\right)^{k} \subseteq I \text { for every irreducible } \mathfrak{m} \text { primary ideal } I\right\} .
\end{aligned}
$$

The second equality follows because every ideal $I$ can be written as an intersection of $\mathfrak{m}$-primary irreducible ideals $I_{n}$, and if we assume that $\left(I_{n}^{*}\right)^{k} \subseteq I_{n}$ for all $n$, it follows that

$$
\left(I^{*}\right)^{k}=\left(\left(\bigcap_{n} I_{n}\right)^{*}\right)^{k} \subseteq\left(\bigcap_{n} I_{n}^{*}\right)^{k} \subseteq \bigcap_{n}\left(I_{n}^{*}\right)^{k} \subseteq \bigcap_{n} I_{n}=I .
$$




\subsection{Definition.}

$$
\begin{gathered}
t(R):=\inf \{k \mid \text { there exists a sequence of tightly closed ideals of type } \\
k \text { that are cofinal with the powers of } \mathfrak{m}\} .
\end{gathered}
$$

We refer to $t(R)$ as "the smallest possible type of $\mathfrak{m}$-primary tightly closed ideals that are cofinal with the powers of $\mathfrak{m} "$, and we show that there is a strong connection between $c(R)$ and $t(R)$.

2.6. Observation. 1. Note that the $\operatorname{ring} R$ is weakly F-regular if and only if $c(R)=1$, and also if and only if $t(R)=1$. Thus, $c(R)$ and $t(R)$ can be thought of as a measure of the failure of the ring to be weakly F-regular.

2. If $R$ is complete and $n$ denotes the number of generators of the test ideal $\tau$ of $R$, Theorems 2.2 and 2.3 above show that $c(R) \leq n$, and also that $t(R) \leq n$. The results of this paper show that $c(R) \leq t(R)$. This result can be thought of as a generalization of the characterization of weakly F-regular rings as those rings for which there exist irreducible tightly closed ideals that are cofinal with the powers of $\mathfrak{m}$.

A different direction, which inspired the results of this paper, is the notion of big ideals introduced in $[\mathrm{HHV}$ :

2.7. Definition. An ideal $J \subset R$ is called big if for every ideal $I \supseteq J, I$ is tightly closed.

The following was proved in $[\mathrm{HHV}$ :

2.8. Theorem. Let $(R, \mathfrak{m})$ be a Noetherian local ring of characteristic $p>0$. Let $\mathfrak{a}$ be an $\mathfrak{m}$-primary irreducible ideal, let $J$ be a tightly closed ideal with $J \supset \mathfrak{a}$ and let $f_{1}, \ldots, f_{n}$ be generators for $(\mathfrak{a}: J) / \mathfrak{a}$, so that we can write $\mathfrak{a}: J=\left(\mathfrak{a}, f_{1}, f_{2}, \ldots, f_{n}\right)$. Then the ideal $\left(\mathfrak{a}, f_{1}, f_{2}, \ldots, f_{n-1}\right): f_{n}$ is big.

We propose the following extension:

2.9. Definition. An ideal $J$ is called $k$-big for some positive integer $k$ if for every $I \supseteq J$, we have $\left(I^{*}\right)^{k} \subseteq I$.

\section{MAin RESUlt}

The following is the main result of this paper:

3.1. Theorem. Let $(R, \mathfrak{m})$ be a Noetherian local ring of characteristic $p>0$. Let $\mathfrak{a}$ be an $\mathfrak{m}$-primary irreducible ideal, let $J$ be a tightly closed ideal with $J \supset \mathfrak{a}$ and let $f_{1}, \ldots, f_{n}$ be generators for $(\mathfrak{a}: J) / \mathfrak{a}$, so that we have $\mathfrak{a}: J=\left(\mathfrak{a}, f_{1}, f_{2}, \ldots, f_{n}\right)$.

Then for every $1 \leq k \leq n$, the ideal $\left(\mathfrak{a}, f_{1}, \ldots, f_{n-k}\right):\left(f_{n-k+1}, \ldots, f_{n}\right)$ is $k$-big.

Proof. Assume by contradiction that $\left(\mathfrak{a}, f_{1}, \ldots, f_{n-k}\right):\left(f_{n-k+1}, \ldots, f_{n}\right)$ is not $k$ big, and let $I \supseteq\left(\mathfrak{a}, f_{1}, \ldots, f_{n-k}\right):\left(f_{n-k+1}, \ldots, f_{n}\right)$ be an ideal for which there exist $x_{1}, \ldots, x_{k} \in I^{*}$, but $x_{1} \ldots x_{k} \notin I$.

Claim: Under the above assumption, there exists an element $d \in R$ such that the following two conditions hold:

(a) $I \subseteq J: d$, and

(b) there exists an $1 \leq i \leq k$ such that $x_{i} \notin J$ : $d$.

If (a) and (b) hold, we immediately get a contradiction, since $x_{i} \in I^{*}$ implies $x_{i} \in(J: d)^{*} \subseteq J^{*}: d$, and thus $d x_{i} \in J^{*}$ for all $1 \leq i \leq k$; while on the other hand, 
(2) implies that $d x_{i} \notin J$ for some $i$, contradicting the assumption that $J$ is tightly closed.

In order to prove the claim, it is enough to choose $d$ such that:

$\left(\mathrm{a}^{\prime}\right) d \in \mathfrak{a}:\left(f_{1}, \ldots, f_{n-k}, I f_{n-k+1}, \ldots, I f_{n}\right)$ and

$\left(\mathrm{b}^{\prime}\right) d \notin \mathfrak{a}:\left(x_{1} f_{n-k+1}, \ldots, x_{k} f_{n}\right)$.

By Matlis duality, $J=\mathfrak{a}:\left(f_{1}, \ldots, f_{n}\right)$, so $J: d=\mathfrak{a}:\left(d f_{1}, \ldots, d f_{n}\right)$. Thus the choice of $d$ as in $\left(\mathrm{a}^{\prime}\right)$ insures that condition (a) in the claim holds, while the choice of $d$ as in ( $\left.\mathrm{b}^{\prime}\right)$ implies that condition (b) in the claim holds. In order to justify that such a choice of $d$ is possible, we need to prove that

$$
\mathfrak{a}:\left(x_{1} f_{n-k+1}, \ldots, x_{k} f_{n}\right) \nsupseteq \mathfrak{a}:\left(f_{1}, \ldots, f_{n-k}, I f_{n-k+1}, \ldots, I f_{n}\right) .
$$

By Matlis duality in the 0 -dimensional Gorenstein $\operatorname{ring} R / \mathfrak{a}$, this is equivalent to proving that

$$
\left(\mathfrak{a}, x_{1} f_{n-k+1}, \ldots, x_{k} f_{n}\right) \nsubseteq\left(\mathfrak{a}, f_{1}, \ldots, f_{n-k}, I f_{n-k+1}, \ldots, I f_{n}\right) .
$$

Assume by contradiction that

$$
\left(\mathfrak{a}, x_{1} f_{n-k+1}, \ldots, x_{k} f_{n}\right) \subseteq\left(\mathfrak{a}, f_{1}, \ldots, f_{n-k}, I f_{n-k+1}, \ldots, I f_{n}\right) .
$$

Let $\bar{R}$ denote the ring $R /\left(\mathfrak{a}, f_{1}, \ldots, f_{n-k}\right)$, and use - to denote the image of an element of $R$ in $\bar{R}$. Equation (1) implies that for all $i, j=n-k+1, \ldots, n$, there exist $a_{i j} \in I$ such that for all $i$ we have

$$
\overline{x_{i-n+k} f_{i}}=\sum_{j=n-k+1}^{n} \overline{a_{i j} f_{j}} .
$$

Thus, the $k \times k$ matrix

$$
\bar{A}=\left(\begin{array}{cccc}
\overline{a_{n-k+1, n-k+1}-x_{1}} & \frac{\overline{a_{n-k+1, n-k+2}}}{a_{n-k+2, n-k+1}} & \ldots & \overline{a_{n-k+1, n}} \\
\ldots & \ldots & \ldots & \overline{a_{n-k+2, n}} \\
\ldots & \ldots & \ldots & \ldots \\
\frac{a_{n-k+2, n-k+2}-x_{2}}{a_{n, n-k+1}} & \overline{a_{n, n-k+2}} & \ldots & \overline{a_{n n}-x_{k}}
\end{array}\right)
$$

annihilates the vector $\left(\bar{f}_{n-k+1}, \ldots, \bar{f}_{n}\right)^{\mathrm{T}}$. Lifting back to $R$, this implies that $\operatorname{det}(A) \in\left(\mathfrak{a}, f_{1}, \ldots, f_{n-k}\right):\left(f_{n-k+1}, \ldots, f_{n}\right)$. Since $\operatorname{det}(A)$ is of the form $x_{1} \ldots x_{k}+$ elements in $I^{k}$, we get

$$
x_{1} \ldots x_{k} \in\left(\left(\mathfrak{a}, f_{1}, \ldots, f_{n-k}\right):\left(f_{n-k+1}, \ldots, f_{n}\right)\right)+I^{k} \subseteq I,
$$

contradicting the choice of $x_{1}, \ldots, x_{k}$.

3.2. Corollary. Let $(R, \mathfrak{m})$ be a Noetherian local ring of characteristic $p>0$. Then $c(R) \leq t(R)$. If, moreover, the ring is complete, or, more generally, if the test ideal commutes with completion, we have $c(R) \leq t(R) \leq \nu(\tau)$.

Proof. Let $t:=t(R)$. In order to establish the first inequality, we need to show that $\left(I^{*}\right)^{t} \subseteq I$ for every $\mathfrak{m}$-primary ideal $I$. According to the definition of $t$, we can choose a tightly closed $\mathfrak{m}$-primary ideal $J \subseteq I$ of type $t$ and we can write $J=\mathfrak{a}:\left(f_{1}, \ldots, f_{t}\right)$ with $\mathfrak{a} \subset J$ an $\mathfrak{m}$-primary irreducible ideal. Choosing $k=t$ in Theorem 3.1 we see that $J$ is $t$-big, and therefore the desired inclusion holds.

The second inequality has been established in 2.6. 


\section{EXAmples}

We would like to investigate the question of whether the inequalities discussed in this paper, namely $c(R) \leq t(R) \leq \nu(\tau)$, are strict in general. There are certainly examples in which $c(R)<\nu(\tau)$; for example, in any ring that has test ideal equal to $\mathfrak{m}$, we have $c(R)=2$, while the number of generators of $\mathfrak{m}$ is in general larger.

Next, we investigate an example in which the strict inequality $t(R)<\nu(\tau)$ holds. Watanabe shows in $\mathrm{Wa}$ that the rings of the form

$$
R=k\left[t, x t^{a}, x^{-1} t^{b},(x+1)^{-1} t^{c}\right] \subset k(x, t)
$$

are F-rational but not F-pure (hence, not weakly F-regular) if $a, b, c$ are positive integers such that $1 / a+1 / b+1 / c<1$, and $k$ is an algebraically closed field of positive characteristic. See also [HH3] for a more elementary approach in the particular case $a=b=c=4$; the following presentation of the ring $R$ is also provided in [HH3]:

$$
R=\frac{k[t, u, v, w]}{\left(t^{8}-u v, t^{4}(v-w)-v w, u(v-w)-w t^{4}\right)} .
$$

The fact that the ring is F-rational implies that $t(R) \leq \operatorname{type}(R)$ (the type of $R$ is equal to the type of the parameter ideals of $R$, which are tightly closed). In order to compute the type of $R$, note that $t, u+v+w$ form a system of parameters for this ring, and using the presentation described above we have $R /(t, u+v+w) \cong$ $k[u, v] /\left(u^{2}, u v, v^{2}\right)$; this is a zero-dimensional ring with socle generated by $u$ and $v$, thus type $(R)=2$. Since the ring is not F-regular, $t(R)>1$. Thus we can conclude that $t(R)=2$.

On the other hand, we claim that the number of generators of the test ideal is at least 3 , thus proving the strict inequality $t(R)<\nu(\tau)$. Note that the test ideal must be m-primary, since $R$ is a two-dimensional normal ring. We need to show that the test ideal cannot be generated by a system of parameters. Assuming the contrary, i.e., that $\tau=(a, b)$ with $a, b$ a system of parameters for $R$, and using the results of $\mathrm{Vr}]$ and $[\mathrm{Hu}]$ (the results of $[\mathrm{Vr}]$ apply since $R$ can be graded by assigning degrees 1 , $4,4,4$ respectively to the variables $t, u, v, w)$, it follows that the ideals $(a): b=(a)$ and $(b): a=(b)$ are big; thus, any ideal that contains either one of $a$ or $b$ must be tightly closed. However, the computation in [HH3] shows that $t^{7} \in(u, v, w)^{*}$, while $t^{7} \notin(u, v, w)$; thus the ideal $I=(u, v, w)$ is not tightly closed. This implies that neither one of $a, b$ is in $I$. We can write $a=\gamma_{1} t^{m}+\alpha$ and $b=\gamma_{2} t^{n}+\beta$ with $\alpha, \beta \in I, \gamma_{1}, \gamma_{2}$ units in $k$. Assuming $m \leq n$, let $b^{\prime}:=b-\gamma_{1}^{-1} \gamma_{2} a t^{n-m} \in I$ and note that $(a, b)=\left(a, b^{\prime}\right)$; therefore the ideal $\left(b^{\prime}\right): a=\left(b^{\prime}\right)$ must also be big, which contradicts the fact that $I$ is not tightly closed.

\section{REFERENCES}

[HH1] M. Hochster and C. Huneke, Tight closure, invariant theory, and the Briançon-Skoda theorem, J. Amer. Math. Soc. 3 (1990), 31-116. MR 91g:13010

[HH2] M. Hochster and C. Huneke, F-regularity, test elements, and smooth base change, Trans. Amer. Math. Soc. 346 (1994), 1-62. MR 95d:13007

[HH3] M. Hochster and C. Huneke, Tight closure of parameter ideals and splitting in modulefinite extensions, J. Algebraic Geom. 3 (1994), 599-670. MR 95k:13002

[HHV] M. Hochster, C. Huneke, and A. Vraciu, Big ideals, in preparation.

$[\mathrm{Hu}]$ C. Huneke, Tight closure and strong test ideals, J. Pure Applied Algebra 122 (1997), 243-250. MR 98g:13003

[Vr] A. Vraciu, Strong test ideals, J. Pure Applied Algebra 167 (2002), 361-373. MR 2003a:13004 
[Wa] K.-i. Watanabe, Study of F-purity in dimension two, Algebraic Geometry and Commutative Algebra in honor of Masayoshi Nagata, vol. II, Kinokuniya, Tokyo, 1988, 791-800. MR 90b:14005

Department of Mathematics, University of Kansas, LaWrence, Kansas 66045

E-mail address: avraciu@math.ukans.edu

Current address: Department of Mathematics, University of South Carolina, Columbia, South Carolina 29208

E-mail address: vraciu@math.sc.edu 